\title{
钯催化的有机嗍化物与羧酸衍生物的交叉偶联反应
}

\author{
杨 军* 邓敏智* 于 涛 \\ (中国科学院上海有机化学研究所 上海 200032)
}

\begin{abstract}
摘要 钯催化的交叉偶联反应是构建 $\mathrm{C}-\mathrm{C}$ 键的常用方法, 近年来, 钯催化的有机硼化物与羧酸衍生物的交叉偶联反 应已成为偶联反应研究中的热点. 本文综述了最近十几年多种有机硼化物与酰氯、酸酐、羧酸、氯甲酸衍生物、羧酸 活性酯、羧酸硫酯的交叉偶联反应研究进展，并对该反应在有机合成中的应用进行了讨论.
\end{abstract}

关键词 羧酸衍生物; 交叉偶联; 钯催化剂

\section{Palladium-Catalyzed Cross-Coupling Reaction of Organoboron Compounds with Carboxylic Derivatives}

\author{
Yang, Jun* Deng, Minzhi* Yu, Tao \\ (Shanghai Institute of Organic Chemistry, Chinese Academy of Sciences, Shanghai 200032)
}

\begin{abstract}
Palladium-catalyzed cross-coupling reaction has emerged as an important strategy to the formation of C-C bond Recently, palladium-catalyzed cross-coupling reaction between organoboron compounds and carboxylic derivatives has become a hotspot of organic chemistry. In this paper, recent researches of organoboron compounds with acyl chlorides, acid anhydrides, carboxylic acids, chloroformic derivatives, carboxylic esters and thiol esters in Suzuki cross-coupling are summarized, and the examples of these reactions in synthesis are also discussed.
\end{abstract}

Keywords carboxylic derivatives; Suzuki cross-coupling; palladium catalyst

碳-碳的形成反应是合成天然产物、药物及其中间 体以及聚合物等的最基本的反应，寻找选择性好，官能 团兼容性好, 反应条件温和的碳-碳键形成方法一直是 有机化学反应研究的热点. 三十多年来通过过渡金属催 化的金属有机化合物与亲电试剂的交叉偶联反应已发 展成为较成熟的方法. 有机硼化物在有机合成中具有重 要作用, 其与卤代亲电试剂的交叉偶联反应，一般被称 作 Suzuki 偶联或 Suzuki-Miyaura 偶联, 是过渡金属催化 的交叉偶联反应中较独特的一种. 有机硼化物的交叉偶 联反应主要优点如下 ${ }^{[1]}$ : 有机硼化物对空气和湿气的耐 受性好, 可被分离、鉴定; 有机嗍化物基本无毒且偶联 产物易于分离; 有机嗍化物的偶联反应条件温和, 官能 团兼容性好; 有机嗍化合物中硼化学键的共价性质仍较 强, 其硼原子含有空 $\mathrm{p}$ 轨道, 在偶联反应中可通过加入 路易斯碱调控反应的活性和选择性.

目前国内外均已有多篇很好的综述对有机嗍的交 叉偶联反应中常见的亲电试剂如卤代芳烃、卤代烯烃的
偶联进行了归纳汇总, 虽然目前文献报道中可与有机硼 化物发生催化偶联反应的羧酸衍生物已有五种以上, 但 相关综述仍然不多、年代较早且能涵盖多种羧酸衍生物 的综述极少. 如 1999年, Dieter ${ }^{[2]}$ 对芳香酰氯与多种金属 有机化合物的偶联反应作了很好的综述, 但由于当时有 机嗍酸与以酰氯为代表的羧酸衍生物的研究刚刚开展, 未被该综述介绍; 2001 年 Yamamoto 等 ${ }^{[3]}$ 的综述仅描述 了 3 例早期关于酸酕和活性酯类化合物两类亲电试剂同 芳基硼酸的偶联; 2003 年 Zapf 等 ${ }^{[4]}$ 的综述侧重于含有氧 羰基的羧酸、酸䣶和活性酯类化合物的偶联; 2007 年 Xin 等 ${ }^{[5]}$ 对苯基硼酸与羧酸衍生物的 Suzuki 类反应, 特别是 水相 Suzuki 类反应进行了介绍. 本文希望能够更全面的 介绍钯催化下有机嗍化物与多种羧酸衍生物及其类似 物(如酰氯、酸䣶、羧酸、活性酯、硫酯、氯甲酸衍生 物等)进行交叉偶联的最新进展, 同时对近期钯催化的 有机嗍化物与羧酸衍生物偶联合成应用的文献进行介 绍.

\footnotetext{
*E-mail: yangj@sioc.ac.cn; dengmz@sioc.ac.cn

Received March 4, 2013; revised April 5, 2013 ; published online April 7, 2013.

Project supported by the National Natural Science Foundation of China (Nos. 20802088, 91017006, 90917017).

国家自然科学基金(Nos. 20802088, 91017006, 90917017)资助项目.
} 


\section{1 有机嗍化物与酰氯的交叉偶联反应}

在䍨酸衍生物与有机嗍化物的交叉偶联反应报道 中, 以酰氯为亲电试剂的反应和合成应用实例较为多 见. 这一方面是因为酰氯一般商品可购, 从羧酸制备相 应酰氯也非常方便, 另一方面是酰氯的氧化加成活性大 大优于芳基氯.

在过渡金属催化的有机嗍化物与羧酸衍生物的偶 联反应报道之前, Negishi 等 ${ }^{[6]}$ 报道了四烷基硼化锂与烷 基酰氯和芳香酰氯的反应: 酰氯和酰溴均可顺利偶联而 酰氟收率极低, 芳香酰氯和烷基酰氯均可以顺利偶联, 该反应的亲核试剂来自有机锂与三烷基硼的加合物官 能团兼容性不高且制备不易. 因此相对比而言，此后报 道的催化偶联反应亲核试剂适用范围较广, 常见有机硼

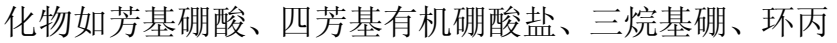
基砋酸等均可以与酰氯发生偶联得到不对称的䣶.

\section{1 有机硼烷与酰氯的交叉偶联反应}

1993 年 Uemura 等 ${ }^{[7]}$ 较早的报道了 $25^{\circ} \mathrm{C}$ 下，四氢呋 喃溶剂中零价钯催化下，四苯硼钠与酰氯可顺利发生交 叉偶联反应得到产物䣶，为了实现高收率的反应，作者 使用了与亲电试剂等当量的四苯嗍钠.

1997 年 Bumagin 等 ${ }^{[8]}$ 对四苯硼钠、苯基硣酸与酰氯 的偶联反应研究表明在无膦配体的条件下钯催化剂可 实现四苯硼钠与三种酰氯的交叉偶联反应，值得注意的 是在该反应条件下一分子四苯嗍钠可与四分子酰氯反 应得到芳基酮衍生物 (Scheme 1).

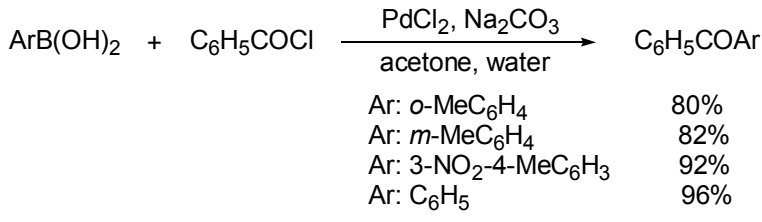

$$
\begin{aligned}
& \begin{array}{ccc}
\mathrm{Ph}_{4} \mathrm{BNa}+4 \mathrm{RCOCl} & \stackrel{\mathrm{PdCl}_{2}}{\mathrm{Na}} 4 \mathrm{RCOPh} \\
\cline { 2 - 3 } & \mathrm{Na}_{2} \mathrm{CO}_{3}, \text { acetone } & \\
& \mathrm{R}: m-\mathrm{NO}_{2} \mathrm{C}_{5} \mathrm{H}_{4} & 66 \% \\
\mathrm{R}:(E)-\mathrm{CH}=\mathrm{CHPh} & 96 \% \\
& \mathrm{R}: \mathrm{Ph} & 96 \%
\end{array}
\end{aligned}
$$

Scheme 1

此后 Bumagin 等 ${ }^{[9]}$ 又进一步报道了醋酸钯在无膦配 体条件催化下四苯嗍钠、苯基嗍酸与多种酰氯在丙酮和 水混合溶剂中的交叉偶联反应, 在干燥的溶剂丙酮中加 入适量的水可将反应时间由 $6 \mathrm{~h}$ 缩短至 $15 \mathrm{~min}$ 而收率仅 轻微下降; 在最优条件下 0.25 当量的四苯硼钠可以与 1 equiv. 的烷基酰氯， $\alpha, \beta$-不饱和酰氯，芳香酰氯和芳杂环 酰氯发生偶联. 作者认为在该反应中四苯砋钠通过逐渐

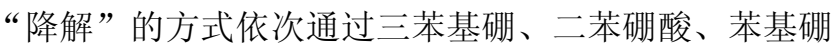
酸的中间体最后生成无机嗍酸 (Scheme 2).

$$
0.25 \mathrm{Ar}_{4} \mathrm{BNa}+\mathrm{RCOCl} \frac{\mathrm{Pd}(\mathrm{OAc})_{2}}{\mathrm{Na}_{2} \mathrm{CO}_{3}, \text { acetone }} \mathrm{RCOAr}
$$

$$
\begin{aligned}
& \text { R: aryl, heteroaryl, cinnamoyl, alkyl } \\
& 14 \text { examples yield 59\% 98\% } \\
& \mathrm{Ph}_{4} \mathrm{BNa} \underset{-\mathrm{PhCOPh}}{\stackrel{\mathrm{PhCOCl}}{-}} \mathrm{Ph}_{3} \mathrm{~B} \underset{-\mathrm{PhCOPh}}{\stackrel{\mathrm{PhCOCl}}{\longrightarrow}} \mathrm{Ph}_{2} \mathrm{BOH} \\
& \underset{-\mathrm{PhCOPh}}{\stackrel{\mathrm{PhCOCl}}{\longrightarrow}} \mathrm{PhB}(\mathrm{OH})_{2} \underset{-\mathrm{PhCOPh}}{\stackrel{\mathrm{PhCOCl}}{\longrightarrow}} \mathrm{H}_{3} \mathrm{BO}_{3}
\end{aligned}
$$

\section{Scheme 2}

随后 Kabalka 等 ${ }^{[10]}$ 以钯催化实现了三烷基硼与酰氯 的偶联，该方法可同时应用于芳基酰氯和烷基酰氯的偶 联反应，当应用环丙基酰氯作为亲电试剂时该偶联反应 收率较低 (Eq. 1).

$$
\begin{array}{r}
\mathrm{R} \mathrm{R}_{\mathrm{Cl}}^{\mathrm{O} \text { Aryl, alkyl, cyclopropyl }} \\
\mathrm{R}^{1} \text { : trialkyl, alkyl }+\mathrm{BBN} \\
\text { yield: } 34 \% \sim 74 \%
\end{array}
$$

2002 年 Wang 等 ${ }^{[11]}$ 在钯催化下, 以 $\mathrm{Al}_{2} \mathrm{O}_{3}$ 负载的 $\mathrm{KF}$ 作碱，通过微波条件实现了芳香酰氯与四苯硼钠的交叉 偶联反应，在微波条件反应 $5 \mathrm{~min}$ 后一分子四苯嗍钠就 可以完成与四分子芳香酰氯的偶联，该反应条件下使用 不同芳香酰氯及肉桂酰氯均以优秀的收率得到芳香 酮 (Eq. 2).

$$
\begin{gathered}
\mathrm{NaBPh}_{4}+4 \mathrm{R}_{\mathrm{Cl}} \frac{\mathrm{PdCl}_{2}\left(\mathrm{PPh}_{3}\right)_{2}, \mathrm{KF} / \mathrm{Al}_{2} \mathrm{O}_{3}}{\text { Acetone, } \mathrm{MW}} 4 \\
\begin{array}{l}
\mathrm{R} \text { : aryl, cinnamoyl } \\
10 \text { examples yield } 90 \% \sim 97 \%
\end{array}
\end{gathered}
$$

2004 年 Hevesi 等 ${ }^{[12]}$ 报道了大位阻三取代烯基硼烷 可与当量铜反应所得到的烯基铜可与各种亲电试剂发 生偶联反应得到三取代的 $\alpha, \beta$-不饱和酮，该反应与钯催 化反应类似. 而当在位阻更大的三取代烯基硼烷反应中 使用了甲基锂后，只得到了甲基亲核试剂与酰氯的偶联 产物 (Scheme 3)

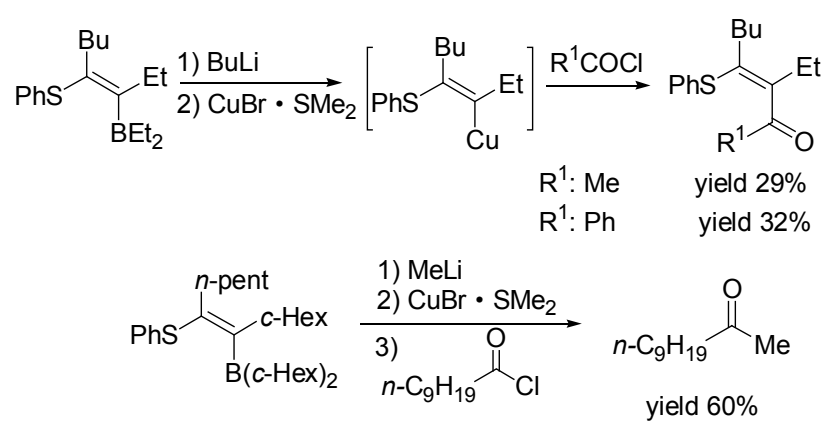

Scheme 3 


\section{2 有机嗍酸与酰氯的交叉偶联反应}

有机嗍酸是目前多种有机嗍化物中制备、合成、使 用和购买最方便的, 因此在有机嗍化物与酰氯的偶联反 应中，使用有机嗍酸作为亲核试剂的报道最多.

1999 年 Haddach 等 ${ }^{[13]}$ 在无水条件下进行碱的改进 实现了钯催化的有机硼酸与酰氯的偶联, 以 $\operatorname{Pd}\left(\mathrm{PPh}_{3}\right)_{4}$ 为催化剂, 5 equiv. 无水碳酸铯作碱可以最高 $80 \%$ 的收率 得到二芳酮, 当应用烷基酰氯作为亲电试剂时该反应也 可以最高 50\%的收率得到 1-芳基-1-烷基酮(Eq. 3).

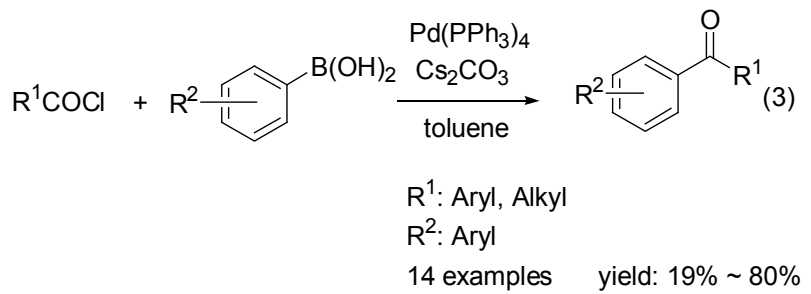

2000 年 Deng 等 ${ }^{[14]}$ 通过氧化银助催化 $\mathrm{PdCl}_{2}$ (dppf) 实现了环丙基硼酸与芳香酰氯的交叉偶联反应，并发现 手性环丙基硣酸在可以顺利的与芳香酰氯偶联，该反应 中发生亲核进攻的碳原子得到了手性保持 (Scheme 4).

$$
\begin{aligned}
& \mathrm{R}^{1} \nabla_{\mathrm{B}(\mathrm{OH})_{2}}{ }^{+} \mathrm{R}^{2} \stackrel{\mathrm{O}}{\mathrm{Cl}} \stackrel{\stackrel{\text { Cat. } \mathrm{PdCl}_{2}(\mathrm{dppf})}{\mathrm{Ag}_{2} \mathrm{O}, \mathrm{K}_{2} \mathrm{CO}_{3}}}{\mathrm{R}^{1}: \text { cyclopropyl }} \\
& \mathrm{R}^{2} \text { : aryl, heteroaryl } \\
& 19 \text { examples yield } 44 \% \sim 78 \%
\end{aligned}
$$

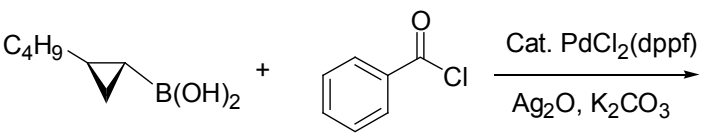

$e e \%=90 \%$

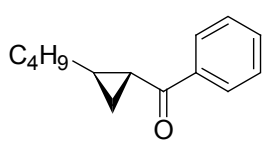

yield $77 \%$, ee $92 \%$

\section{Scheme 4}

2006 年 Polackova 等 ${ }^{[15]}$ 详细研究了微波条件下钯催 化的芳基硼酸与酰氯的交叉偶联反应, 在 $\operatorname{Pd}\left(\mathrm{PPh}_{3}\right)_{4}$ 催 化下以碳酸铯作碱条件下使用微波反应可实现多种芳 香酰氯的交叉偶联反应，烷基酰氯和月桂酰氯在该条件 下不反应 (Eq. 4).

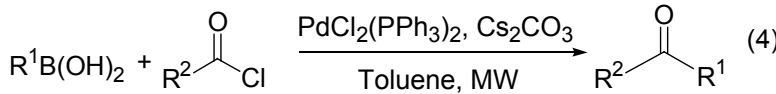

$$
\begin{aligned}
& \mathrm{R}^{1} \text { : aryl } \\
& \mathrm{R}^{2} \text { : aryl, heteroaryl } \\
& 19 \text { examples yield } 18 \% \sim 93 \%
\end{aligned}
$$

2003 年 Urawa 等的研究结果表明在 $\alpha, \beta$-不饱和酰氯 与芳基硼酸的偶联反应中水合磷酸钾是最适用的碱，作 者认为水合磷酸钾中的结晶水显著的活化了催化剂 ${ }^{[16]}$, 烯基酰氯、芳基酰氯和烷基酰氯都能以中等到良好的收 率得到偶联产物 ${ }^{[17]}$ (Eq. 5).

$$
\begin{aligned}
& \begin{array}{c}
\mathrm{R}^{1} \text { : methyl, aryl } \\
\mathrm{R}^{2} \text { : methyl } \\
\mathrm{R}^{3} \text { : methyl } \\
\mathrm{R}^{4} \text { : aryl, heteroaryl } \\
9 \text { examples yield } 7 \% \sim 99 \%
\end{array} \\
& \begin{array}{l}
\mathrm{R}^{3} \\
\text { toluene }
\end{array}
\end{aligned}
$$

同时作者对于芳基硼酸酯和芳基硼酸酎(boroxine) 的偶联进行了探索, 在上述最优条件下两种有机硼化物 均可顺利与 2-methylbut-2-enoyl chloride 发生偶联反应 得到相应的 $\alpha, \beta$-不饱和酮(Scheme 5).
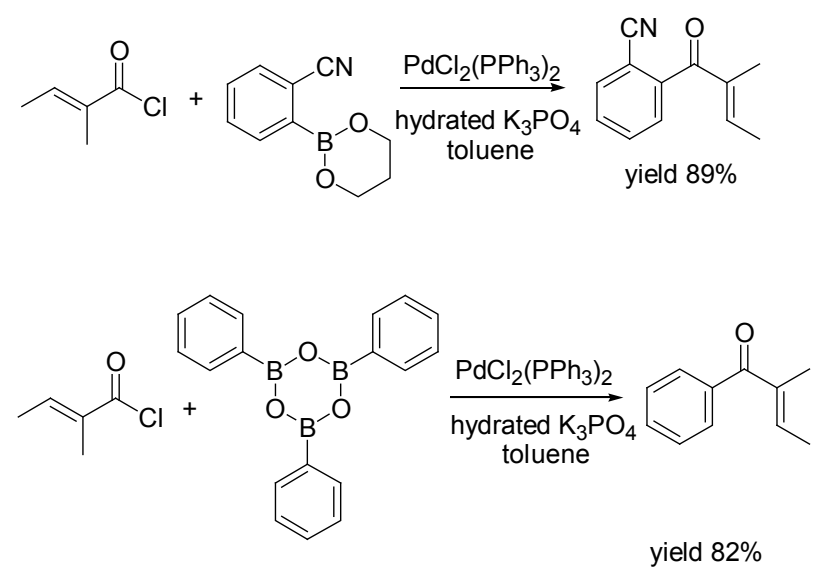

Scheme 5

\section{3 有机硼酸与酰氯的交叉偶联反应新条件研究}

随着偶联反应中钯催化剂和配体研究的深入，在有 机嘲酸与酰氯交叉偶联反应中探索新的催化剂、配体、 溶剂、新的反应方法和氧化条件等也成为该偶联反应研 究的一个重点.

2005 年 Bandgar 等 ${ }^{[18]}$ 报道一例无膦配体条件下氯 化钯催化的有机磀酸与酰氯的固相交叉偶联, 在最优条 件下芳香酰氯可以与芳基硼酸(14 例，收率 78\% 95\%) 甚至烷基硼酸(异丙基硼酸，收率 90\%)顺利的发生交叉 偶联反应, 而当酰氯 $\alpha$ 碳为 $\mathrm{sp}^{3}$ 杂化碳原子时四种酰氯 均不能偶联.

2005 年 Nishihara 等 ${ }^{[19]}$ 在钯催化的芳基硼酸与酰氯 偶联反应中引入了第二金属噻吩-2-甲酸亚铜 $(\mathrm{CuTc})$ 并 研究了其对于交叉偶联反应的影响，其研究结果表明当 量的噻吩-2-甲酸亚铜对该反应有明显的促进作用，可 
能的机理是反应过程中氧化加成生成的羰基氯化钯与 芳基嗍酸和噻吩-2-甲酸亚铜生成了较稳定的六元环过 渡态(Scheme 6), 从而促进了转金属反应在室温下顺利 进行, 室温下使用乙醚作溶剂被认为可避免副产物的生 成. 该反应底物拓展结果表明在室温下 $3 \mathrm{~h}$ 内各种取代 的芳基硼酸即可以中等到优秀的收率完成与芳基酰氯、 杂芳基酰氯、烷基酰氯和肉桂酰氯的偶联反应.

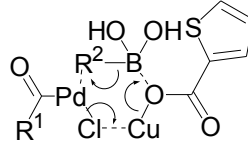

\section{Scheme 6}

2006 年 Zhang 等 ${ }^{[20]}$ 报道了在水相中催化的芳香酰 氯和酸䣶与芳基硼酸的交叉偶联反应，在水中加入聚乙 烯醇或离子液体后, 醋酸钯可作为钯源不需添加膦配体 即可完成反应，其中催化剂-水相-离子液体可以循环使 用.

2008 年 Wolf 等 ${ }^{[21]}$ 制备了二(二叔丁基次磷酸)氯化 钯，以其催化的酰氯与芳基硼酸的交叉偶联反应具有良 好的底物适用性，此外当使用微波条件时反应时间缩短 为 $10 \mathrm{~min}$.

2011 年 $\mathrm{Li}$ 等 ${ }^{[22]}$ 分离并鉴定了含有新型 $\mathrm{N}$-杂环卡宾 配体的钯催化剂, 并使用该催化剂完成了 15 例芳基酰 氯与芳基硼酸的交叉偶联.

2011 年 Aguiar 等 ${ }^{[23]}$ 开展了负载型非均相钯催化剂 和均相钯催化剂催化的芳基硼酸与芳香酰氯的反应进 行了对比, 并对这一反应中脱羰偶联得到联苯衍生物的 问题进行了分析.

2012 年 $\mathrm{Wu}$ 等 ${ }^{[24]}$ 将二茂铁亚胺环钯催化剂应用于 芳基硼酸与芳香酰氯及芳香酸䣶的交叉偶联反应后发 现, 该催化剂活性较高, 其催化剂经八次循环使用后仍 可催化酰氯或酸䣶的交叉偶联反应达到 $90 \%$ 的收率; 2012 年 Chen 等 ${ }^{[25]}$ 研究了钯催化空气作为氧化剂的芳基 嗍酸与芳香酰氯的交叉偶联反应，该偶联条件下获得了 酯类产物而不是常见的酮类产物; 通过 ${ }^{18} \mathrm{O}$ 标记实验证 实了酯基中新的氧原子来自氧气(Scheme 7).

\section{4 有机嗍酸与酰氯的交叉偶联反应在合成中的应用}

通过羧酸衍生物与金属有机化合物的反应制备酮 类化合物是碳一碳键构建和官能团转化中的重要反应. 金属有机化合物与羧酸衍生物的 1,2-加成反应所得酮产 物还会进一步接受亲核进攻形成叔醇产物; 过渡金属催 化下使用有机嗍等金属有机化合物与羧酸衍生物的偶 联反应选择性好、条件温和且官能团兼容性好，目前已 有较多合成中应用实例。

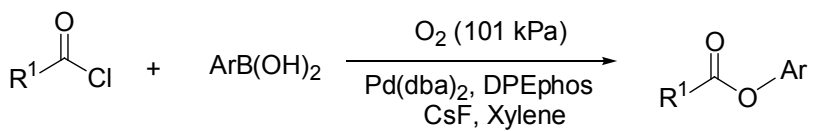

$$
\begin{aligned}
& \mathrm{R}^{1} \text { : aryl, heteroaryl, alkyl, cinnamoyl } \\
& 36 \text { examples yield } 61 \% \sim 97 \%
\end{aligned}
$$

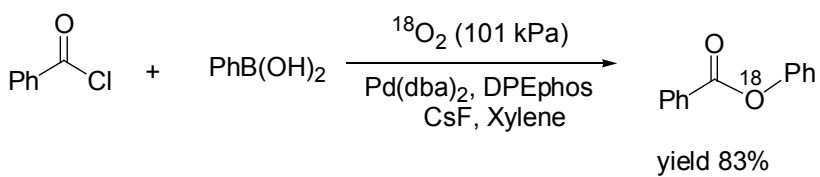

\section{Scheme 7}

2003 年 Rolando 等 ${ }^{[26]}$ 研究了将新的钯催化的有机 嗍化物与酰氯的偶联反应应用于查尔酮衍生物的合成, 比较了苯乙烯基硼酸与芳基酰氯的偶联和苯乙烯基酰 氯与芳基嗍酸的偶联(Scheme 8), 当使用 Bumagin 和 Haddach 报道的条件时，偶联反应收率并不理想; 而改 变合成策略使用芳基酰氯和烯基硼酸进行偶联后以 $81 \%$ 收率得到查尔酮衍生物。

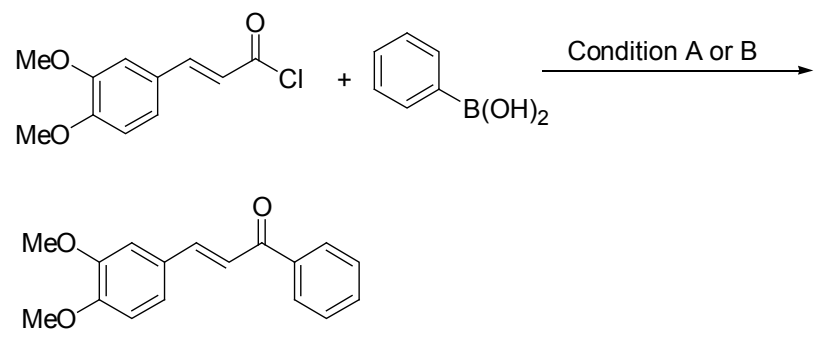

Condition A: Bumagin method, 23\%

Condition B: Haddach method, 41\%

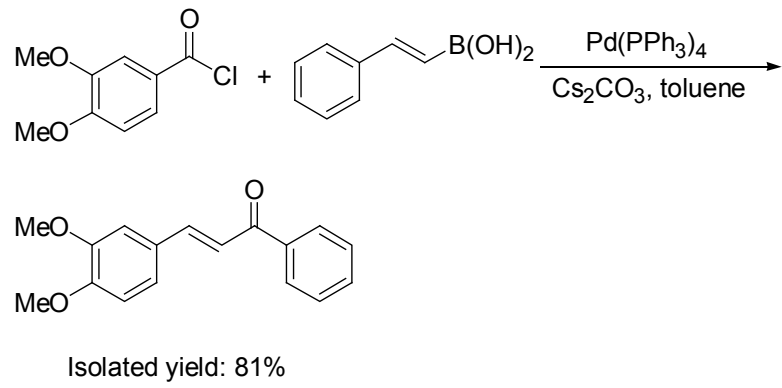

\section{Scheme 8}

同在 2003 年 Lowe 等 ${ }^{[27]}$ 将钯催化的芳基硼酸与芳 基酰氯的交叉偶联反应应用于甘氨酸转运蛋白 NPTS 消 旋体的合成(Scheme 9), 并通过大鼠放射摄取实验测得 NPTS 消旋体的 GLY IC 50 为 $153 \mathrm{~nm}$.

2004 年 Langstrom 等 ${ }^{[28]}$ 研究了钯催化的噻吩-2-硼 酸与多种芳香酰氯的偶联反应，此外通过四苯硼钠与 2萮基酰氯偶联得到了非同位素标记对照品 2'-苯基荟酮. 
<smiles>Cc1ccc([18O]c2ccc(C)cc2)cc1</smiles><smiles>Cc1cccc(C(=O)c2ccc(OC(CCN(C)CC(=O)O)c3ccccc3)cc2)c1</smiles>

Scheme 9

2005 年 Guy 等 ${ }^{[29]}$ 从芳基硼酸出发两步合成了缩氨 基硫脲衍生物, 并评估了其对半胱氨酸蛋白酶的抑制作 用(Scheme 10), 当 $\mathrm{R}$ 为丁基时其缩氨基硫脲衍生物用量 $3 \mu \mathrm{mol} / \mathrm{L}$ 时治愈了克氏锥虫感染的巨噬细胞; 此后 Guy 等 ${ }^{[30]}$ 进一步研究表明这些化合物具有良好膜穿透性和 低毒性，其作用于非洲锥虫病的机理还需进一步研究.
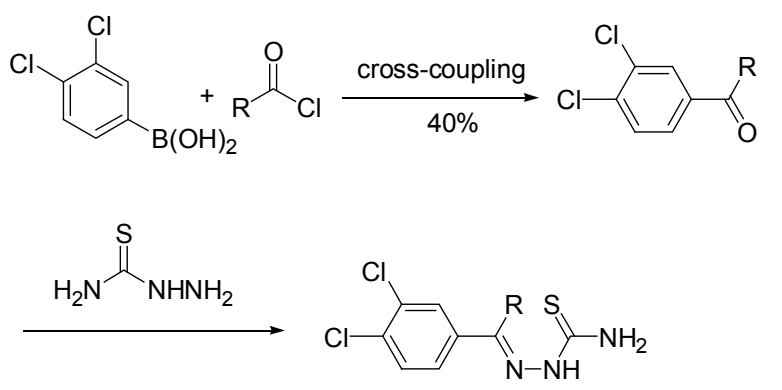

R: alkyl, alkenyl, aryl

\section{Scheme 10}

2006 年 Jacobi 等 ${ }^{[31]}$ 开展了 furanosteroids 天然产物 viridin 模板化合物的合成研究, 采用了现场三步连续反 应构建硼酸片呐醇酯、脱除酯基保护和钯催化芳基硼酸 与酰氯偶联的方法以平均每步 $80 \%$ 的收率得到了合成 的重要中间体(Scheme 11), 并在此基础上进一步完成 了 Viridin 模板化合物的合成.

2009 年 Ambroise 等 ${ }^{[32]}$ 合成了 4 种 3-biphenyl-40-yl5,6-dihydroimidazo[2,1-b]thiazole 化合物, 并以其作为光 化学反应探针研究了甲状腺细胞中碘的流失; 4 种探针 化合物中仅二芳酮类化合物(Target 4, Scheme 12)具有 提高甲状腺细胞内碘含量的生理活性, 该分子由钯催化<smiles>CC(C#CS(C)(=O)=O)c1ccccc1B1OC(C)(C)C(C)(C)O1</smiles>

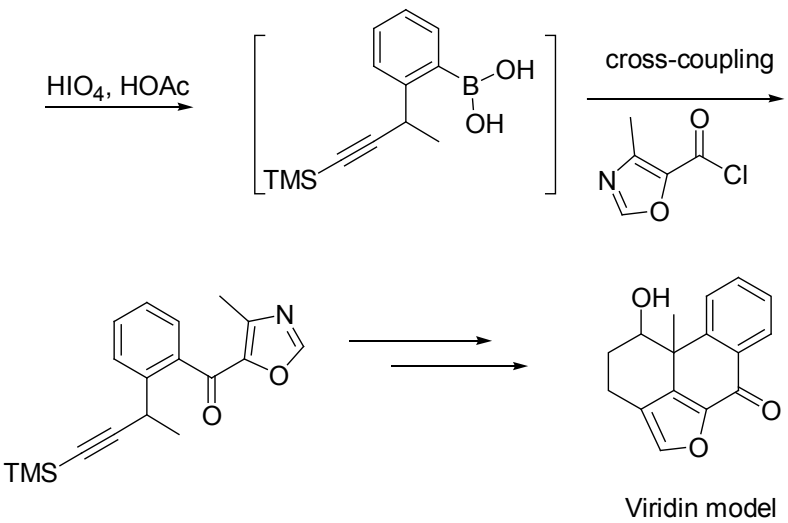

Scheme 11<smiles>CC(=O)c1ccc(O[Se]c2ccc(C(=O)Cl)cc2)cc1</smiles><smiles>CC(=O)c1ccc(C(=O)c2ccccc2)cc1</smiles><smiles>[R]C1=C(c2ccc(C(=O)c3ccccc3)cc2)N2CCN=C2S1</smiles>

R: $\mathrm{H}$, Target 4

R: ${ }^{3} \mathrm{H}$, Labelled Target 4

\section{Scheme 12}

酰氯与对乙酰基苯基硼酸偶联等反应制备, 并可进一步 衍生制备同位素気标记的 Target 4.

2012 年 $\mathrm{Chu}$ 等 ${ }^{[33]}$ 对 FLAP 抑制剂中的降冰片片段 的改进了研究，其中通过钯催化芳基硼酸与特戊酰氯偶 联等反应制备的叔丁基衍生物生理活性优于其它十二 种衍生物(Scheme 13). 该化合物有望作为一种新药物 通过抑制白三烯(leukotriene)的合成来治疗过敏性鼻炎 和哮喘.

2012 年 Keenan 等 ${ }^{[34]}$ 设计并合成三芳基醇化合物并 对其抑制实验鼠血液中雉虫含量进行了测试，其关键化 合物二芳酮可通过钯催化的邻氯苯酰氯与对氯苯基硼 酸的偶联合成(Scheme 14).

2012 年 Yang 等 ${ }^{[35]}$ 报道了一种利用 1,2-噁硼-2(5H)2-醇与芳基酰氯的交叉偶联反应合成多取代呋喃类化 合物的方法，当使用含有羟甲基的顺式烯基硼作为亲核 
<smiles>COC(=O)c1ccc(OCc2ccc3ccccc3n2)cc1C1(c2ccccc2)CC2CCC1C2</smiles>

FLAP inhibitor
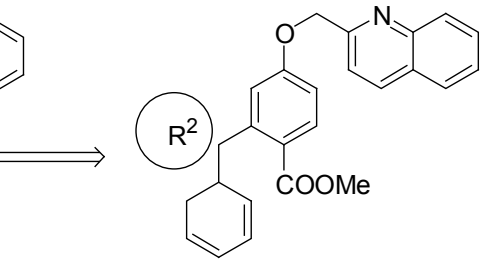

New FLAP inhibitor

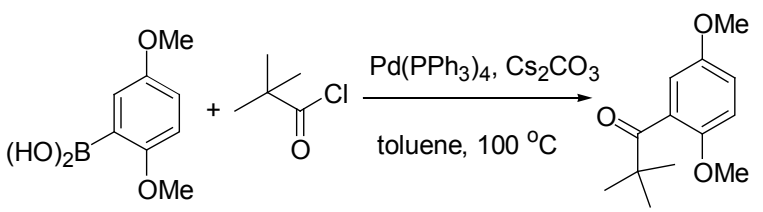<smiles>C#C[C@H](C)C(c1ccc(O)cc1O)C(c1ccccc1)C(C)(C)C</smiles>

Scheme 13<smiles>O=C(Cl)c1ccccc1Cl</smiles>

Cross-coupling reaction or Frediel-Crafts reaction or $\mathrm{S}_{\mathrm{N}} 2$ of aryllithium<smiles>O=C(c1ccc(Cl)cc1)c1ccccc1Cl</smiles>

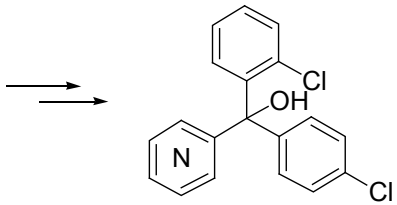

Scheme 14

试剂所得偶联产物可关环以良好到优秀的收率得到 3,4二取代和 2,3,4-三取代呋喃, 这一例有机嗍化合物与酰 氯的偶联反应是合成多取代呋喃类化合物的简便实用 方法(Eq. 6).

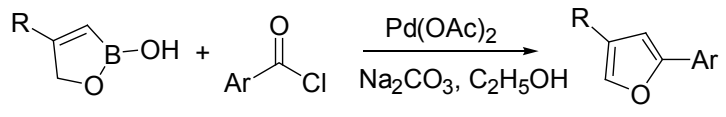

$\mathrm{R}=\mathrm{alkyl}$, aryl

14 examples, $80 \% \sim 93 \%$

\section{2 有机硼化物与酸酐的交叉偶联反应}

2001 年 Gooßen 等 ${ }^{[36]}$ 首次报道了钯催化的有机硼化 物与酸䣶的交叉偶联, 当使用醋酸钯和三邻甲氧基苯基 膦催化下, 各种芳基嗍酸与芳基酰氯可以顺利发生偶联 反应，除了特成酸䣶外大部分烷基酰氯也可以在该条件 发生偶联反应(Eq. 7).

$$
\begin{aligned}
& \text { toluene } \\
& \text { 11 alkyl, alkenyl, phenyl } \\
& \mathrm{R} t \text {-butyles, yield } 91 \% \sim 98 \% \text { yield } 0 \%
\end{aligned}
$$

与普通的 Suzuki 偶联不同，这类钯催化的有机硼酸 与酸酐的交叉偶联反应往往不必使用碱 ${ }^{[36]}$, 且收率高 于此后报道的铑催化的芳基硣酸与酸酐偶联反应 ${ }^{[37]}$, 或许是因为铑催化的反应易得到偶联与脱羰偶联两种 产物 ${ }^{[38]} .2002$ 年 Yamamoto 等 ${ }^{[39]}$ 在其钯催化酸酐制醛的 基础上详细报道其关于钯催化的有机硼化物与酸酐的 交叉偶联的研究结果并提出了可能的机理(Scheme 15); 此外作者对混酐的研究表明芳香基团偶联活性优于叔 丁基，同时使用硫酸钡负载的钯催化剂获得了 $94 \%$ 的产 物.

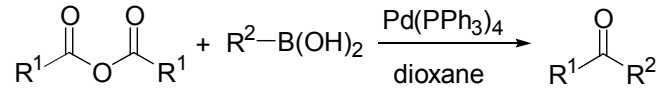

$$
\begin{aligned}
& \mathrm{R}^{1} \text { : aryl } \\
& \mathrm{R}^{2} \text { : aryl, heteroaryl, alkenyl } \\
& 8 \text { examples, yield } 44 \% \sim 96 \%
\end{aligned}
$$

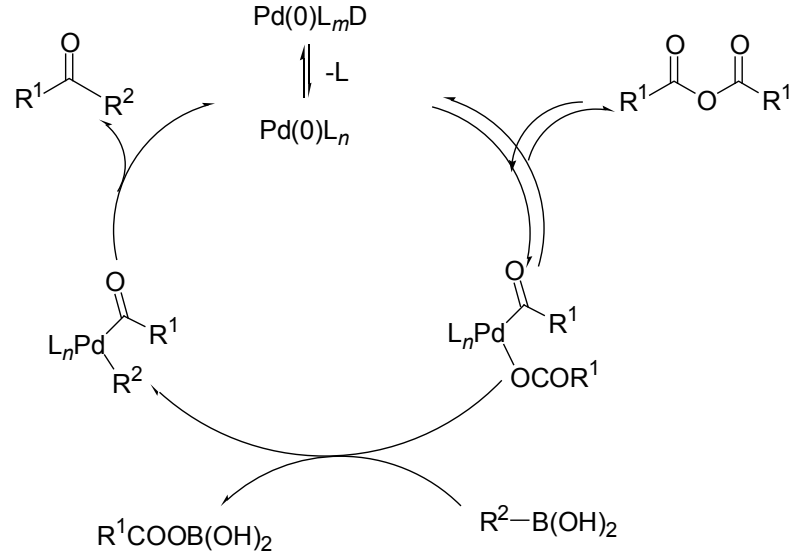

Scheme 15

2004 年 Goossen 和 Thiel 等 ${ }^{[40]}$ 通过计算研究了钯催 化的芳基硼酸与酸酐的交叉偶联计算化学研究，将配体 简化为三甲基膦后，密度泛函理论计算结果表明钯催化 的苯基硼酸与乙酸酐的偶联可能存在两种途径: $\mathrm{Pd}\left(\mathrm{PMe}_{3}\right)_{2}$ 中性催化循环和 $\left[\mathrm{Pd}\left(\mathrm{PMe}_{3}\right)_{2} \mathrm{OAc}\right]^{-}$负离子催化 循环, 作者认为 $\left[\mathrm{Pd}\left(\mathrm{PMe}_{3}\right)_{2} \mathrm{OAc}\right]^{-}$与亲电试剂具有更强 的结合能力. 此后 Goossen 和 Thiel 进一步计算了两种负 离子钯 $\left[\mathrm{Pd}\left(\mathrm{PMe}_{3}\right) \mathrm{OAc}\right]^{-}$和 $\left[\mathrm{Pd}\left(\mathrm{PMe}_{3}\right)_{2} \mathrm{OAc}\right]^{-}$启动的催化 循环, 作者认为上述两种负离子钯催化剂在氧化加成后 均形成 $\left[\mathrm{Pd}\left(\mathrm{PMe}_{3}\right)(\mathrm{OAc})_{2}\right]^{-}$(Scheme 16). 作者在计算了 5 


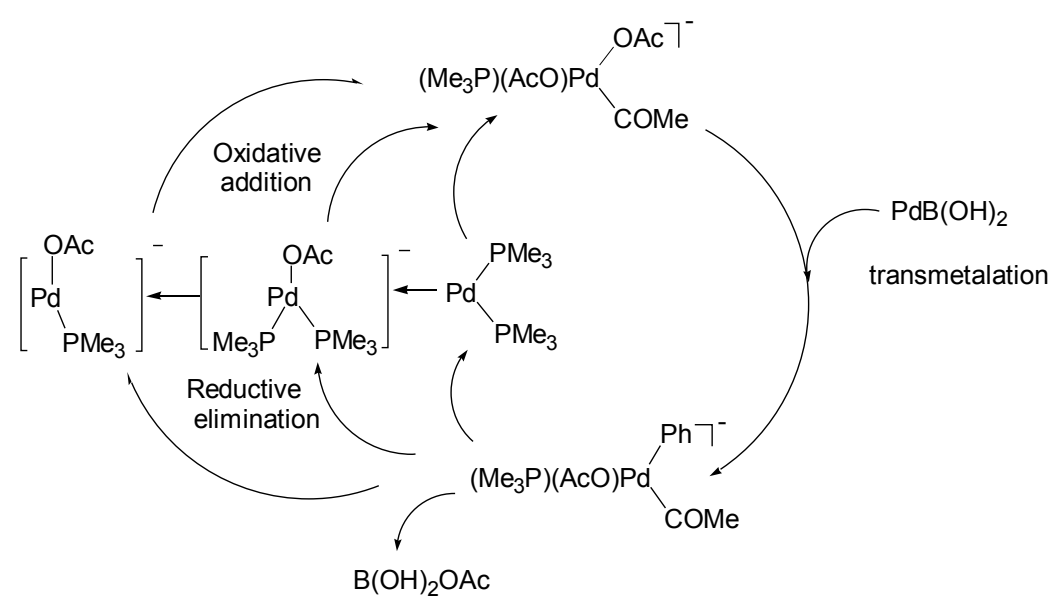

Scheme 16

种中性及负性的催化循环后认为: 从 $\Delta G_{\max }$ 计算结果看 启动的催化循环 $\left[\mathrm{Pd}\left(\mathrm{PMe}_{3}\right)_{2} \mathrm{OAc}\right]^{-}$的计算值为 32.2 $\mathrm{kJ} / \mathrm{mol},\left[\mathrm{Pd}\left(\mathrm{PMe}_{3}\right) \mathrm{OAc}\right]^{-}$启动的催化循环的计算值为 $37.3 \mathrm{~kJ} / \mathrm{mol}$, 两者均显著低于中性催化循环( $82.5 \sim 98.0$ $\mathrm{kJ} / \mathrm{mol})^{[41]}$.

2004 年 Wang 等 ${ }^{[42]}$ 报道了微波促进的四苯硼钠与 硼酸䣲的交叉偶联，该方法将反应时间大大缩短至 12 $\min ($ Eq. 8).

$\mathrm{NaBPh}_{4}+\mathrm{R}_{\mathrm{O}} \stackrel{\mathrm{O}}{\stackrel{\mathrm{O}}{\mathrm{PdCl}_{2}\left(\mathrm{PPh}_{3}\right)_{2}}} \underset{\mathrm{THF}, \mathrm{MW}, \mathrm{N}_{2}}{\stackrel{\mathrm{O}}{\mathrm{O}}}$

R: aryl, alkenyl, Me 10 examples, yield $82 \% \sim 92 \%$

2006 年 Kim 等 ${ }^{[43]}$ 对钯催化的羧酸磷酸䣶与有机 嗍、有机锡的分子间偶联和分子内的 Heck 偶联, 当使 用醋酸钯、三苯基膦和碳酸钠时，一些大位阻的羧酸磷 酸酎如环已基、叔丁基均可顺利偶联得到相应的酮 (Eq. 9).

$$
\begin{aligned}
& \mathrm{R}^{1} \stackrel{\mathrm{O}_{\mathrm{O}}-\mathrm{P}_{\mathrm{OMe}}^{\mathrm{O}-\mathrm{OMe}}}{\mathrm{O}}+\mathrm{R}^{2}-\mathrm{B}(\mathrm{OH})_{2} \frac{\mathrm{Pd}(\mathrm{OAc})_{2}, \mathrm{PPh}_{3}}{\mathrm{Na}_{2} \mathrm{CO}_{3}} \underset{\mathrm{R}^{1}}{\stackrel{\|_{1}}{\longrightarrow}} \mathrm{R}^{2}(9) \\
& \mathrm{R} \text { : aryl, benzyl, } c \text {-hex, } t \text {-Bu } \\
& 16 \text { examples, yield } 50 \% \sim 92 \%
\end{aligned}
$$

2007 年 Zhang 等 ${ }^{[4]}$ 报道了表面活性剂对于水相中 钯催化芳基硼酸与酸酕或酰氯偶联的促进作用, 研究表 明在水相的硼酸与酸酲的偶联反应中 0.5 equiv. 的表面 活性剂即可显著促进反应的进行, 当使用十二烷基苯磺 酸钠(SDS) 时结果最佳, 该反应体系同样适用于钯催化 芳基硼酸与酰氯的偶联反应. 2008 年 Xin 等 ${ }^{\left[{ }^{[5]}\right]}$ 研究了于 水相中钯催化芳基硼酸与酸酐的交叉偶联反应, 该反应 只需使用 $\mathrm{PdCl}_{2}$ 催化剂和水与丙酮的混合溶剂即可在温 和条件下快速完成偶联反应.
钯催化剂与卡宾配体形成的络合物对多种金属试 剂的交叉偶联反应有良好的催化活性，2011 年 Shao 等 ${ }^{[46]}$ 从脯氨酸合成并鉴定了一个新的 NHC-Pd(II)-Im 配 合物(Scheme 17, complex 1), 以其为催化剂在弱碱条件 下可很好的完成芳基硼酸与酸酐的交叉偶联反应; 2012 年 $\mathrm{Lu}$ 等 ${ }^{[47]}$ 报道了另一种 NHC-Pd(II)-Im 配合物(Scheme 17, complex 2), 该配合物可以在纯水溶剂中实现芳基硼 酸与酸䣶的交叉偶联反应.

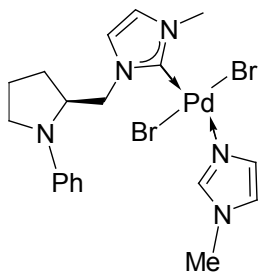

NHC-Pd(II)-Im complex 1

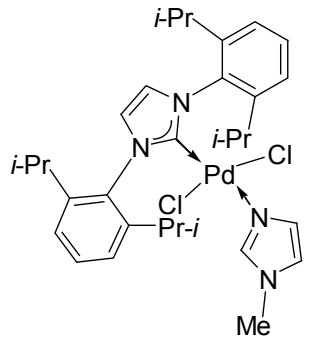

NHC-Pd(II)-Im complex 2

\section{Scheme 17}

\section{3 有机嗍化物与羧酸的交叉偶联反应}

目前文献中还未见钯催化的羧酸与有机硼酸的直 接偶联得到酮的反应, 当前最便捷的策略是将羧酸与活 化剂反应生成酯、酸酐或酸酐类似物后再发生偶联反应.

前已述及在 Gooßen 所报道钯催化的芳基硼酸与酸 酐的偶联条件下特戊酸䣶是不能反应的(Eq. 7), Gooßen 等 ${ }^{[48]}$ 巧妙的利用这一特点发展了将羧酸与偶联惰性特 戊酸酐预反应再偶联的便捷方法, 从而实现了羧酸与有 机嗍化合物的一锅法高效偶联; 在随后的研究中 Gooßen 等 ${ }^{[49]}$ 发现二碳酸二甲酯(dimethyl dicarbonate)也 可以替代叔丁酸䣶用于钯催化的芳基硼酸与酸酐的偶 联, 通过在反应体系中加入 2.5 equiv.的水并控制单膦 配体：钯的比例 $<4: 1$ 后可以在较低温度下获得良好 的收率(Scheme 18). 

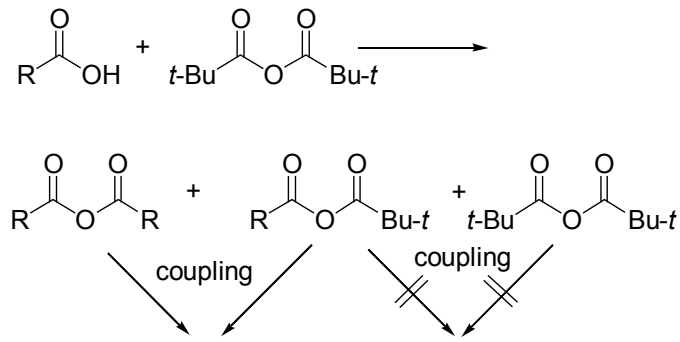

$\stackrel{\mathrm{O}}{\mathrm{Or}}_{\mathrm{Ar}}$

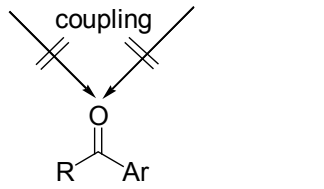

$$
\begin{aligned}
& \mathrm{R}^{1} \mathrm{OH}_{\mathrm{OH}}+\mathrm{R}^{2}-\mathrm{B}(\mathrm{OH})_{2} \frac{(t-\mathrm{BuCO})_{2} \mathrm{O}}{\mathrm{Pd} \text { cat. cross-coupling } \mathrm{R}^{1}} \\
& \begin{array}{l}
\mathrm{R}^{1} \text { : aryl, alkyl } \\
\mathrm{R}^{2} \text { : aryl, heteroaryl } \\
18 \text { examples, yield } 47 \% \sim 90 \%
\end{array}
\end{aligned}
$$

\section{Scheme 18}

几乎同时 Yamamoto 等 ${ }^{[50]}$ 也报道羧酸现场活化制备 酸酐再进一步偶联的方法学, 通过活化剂篮选发现二碳 酸二甲酯作为活化剂效果最好. 该体系可应用于二羧酸 的偶联, 该法也可应用于四苯硼钠与羧酸的偶联, 当使 用羰基羧酸作为亲电试剂时以良好的收率得到了脱羰 偶联的产物。

2001 年 Gooßen 等 ${ }^{[51]}$ 发现 $N$-benzoyloxysuccinimide 可与芳基嗍酸发生交叉偶联反应后高收率的得到芳基 酮产物. 于是作者利用 $\operatorname{di}(N$-succinimidyl $)$ carbonate (DSC)与羧酸结合得到酸基琥珀酰亚胺，再与芳基硼酸 发生偶联反应，“一锅法” 得到偶联产物芳基酮(Eq. 10). 反应中的羧酸既可以是芳基甲酸，也可以是烷基羧酸.

2010 年 Yoon 等 ${ }^{[52]}$ 发展了一种通过 EEDQ 活化羧酸 与芳基硼酸发生交叉偶联的方法(Scheme 19), 在添加 2 equiv. 水后可以良好的收率完成芳基硼酸与各种酰氯的 交叉偶联.

$$
\begin{aligned}
& \mathrm{Ar}-\mathrm{B}(\mathrm{OH})_{2}+\mathrm{R}_{\mathrm{OH}} \stackrel{\mathrm{O}}{\longrightarrow} \mathrm{Ar}_{\mathrm{R}} \\
& \text { Pd cat. } \\
& \mathrm{R}=\mathrm{alkyl}, \mathrm{aryl} \\
& 24 \text { examples, yield } 42 \% \sim 95 \%
\end{aligned}
$$

特殊的羧酸底物还可以发生钯催化的脱羰偶联. 2011 年, Liu 等 ${ }^{[53 a]}$ 报道了钯催化的芳基羧酸与芳基硼酸 及芳基嗍酸酯的偶联反应; 同年, Ge 等 ${ }^{[53 b, 53 c]}$ 先后报道 钯催化的芳基氟硼酸钾与 $\alpha$-酮酸、草酸衍生物的脱羧偶 联. 不同形态的有机硼化物均能与羧酸发生偶联，以中 等到优秀的收率得到脱羰偶联产物(Scheme 20).

\section{4 有机硼化物与氯甲酸衍生物的交叉偶联反应}

在合成化学中氯甲酸酯、氯甲酰胺多用于羟基官能 才的保护及制备酸酐. 而由于氯甲酸基团与酰氯基团的 结构相似，因此不难推断这类化合物也可以应用于偶联 反应中作为亲电试剂.

2005 年 Deng 等 ${ }^{[54]}$ 的研究结果表明在钯催化的有机 硼酸与氯甲酸酯和氯甲酰胺的偶联中, $\mathrm{Cu}_{2} \mathrm{O}$ 对于提高 产物收率有较显著的作用. 在加入催化量的 $\mathrm{Cu}_{2} \mathrm{O}$ 和当 量的 $\mathrm{K}_{3} \mathrm{PO}_{4} \cdot 3 \mathrm{H}_{2} \mathrm{O}$ 后芳基硼酸可以同氯甲酸酯和氯甲酰 胺偶联得到增加 1 个碳原子的酯或酰胺 (Scheme 21). 稍后 Kristensen 等 ${ }^{[55]}$ 报道了类似的芳基硼酸酯与氯甲酰 胺的交叉偶联反应.

2007 年 Takemoto 等 ${ }^{[56]}$ 采用一锅法的策略通过钯催 化的烷基 9-BBN 与氯甲酰胺的交叉偶联反应得到了 $\beta$ 取代丙酰胺，该反应同样适用于芳基硼酸、苯乙烯基硼 酸和三乙基硼等亲核试剂与氯甲酰胺的交叉偶联反应 (Scheme 22).
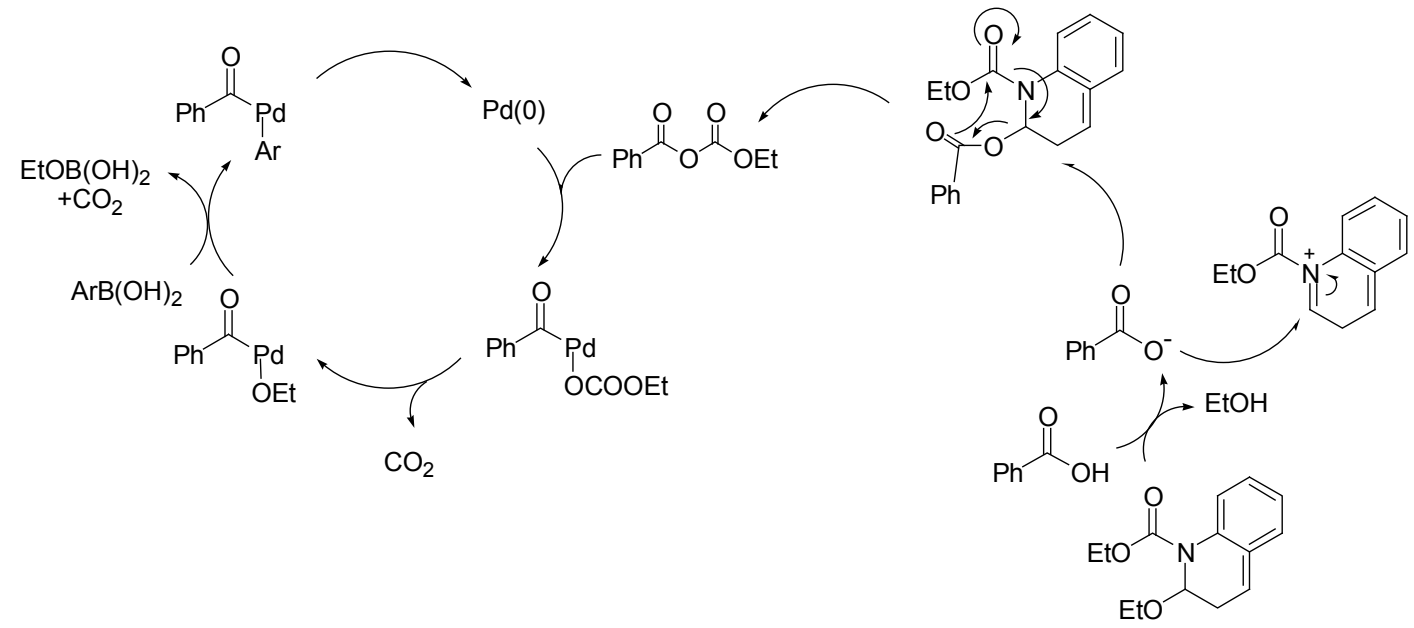

Scheme 19 


$$
\begin{aligned}
& \mathrm{Ar}^{1}-\mathrm{COOH}+\mathrm{Ar}^{2}-\mathrm{BX}_{2} \frac{\mathrm{Pd}(\mathrm{TFA})_{2}, \mathrm{Ag}_{2} \mathrm{CO}_{3}}{\mathrm{DMSO}} \mathrm{Ar}^{1}-\mathrm{Ar}^{2}+\mathrm{CO}_{2} \\
& \mathrm{BX}_{2}=\mathrm{B}(\mathrm{OH})_{2} \quad 18 \text { examples, } 32 \% \sim 97 \% \text { yield } \\
& \mathrm{BX}_{2}=\mathrm{B}_{\mathrm{O}}^{\mathrm{O}} \backslash 23 \text { examples, 32\% 98\% yield }
\end{aligned}
$$

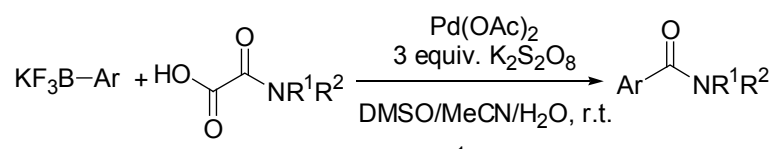

$$
\begin{aligned}
& \mathrm{R}^{1}=\text { alkyl, } \mathrm{H} \\
& \mathrm{R}^{2}=\text { alkyl } \\
& 20 \text { examples, yield } 46 \% \sim 92 \%
\end{aligned}
$$

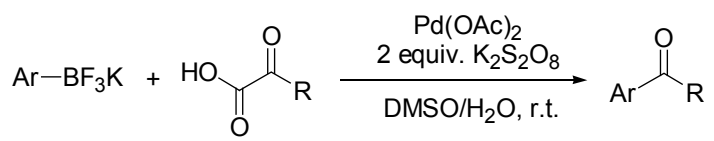

$$
\begin{aligned}
& \mathrm{R}^{1}=\text { aryl, alkyl } \\
& 29 \text { examples, yield } 41 \% \sim 98 \%
\end{aligned}
$$

Scheme 20

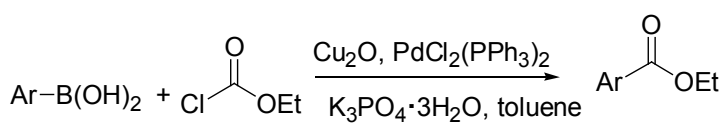

8 examples, yield $21 \% \sim 78 \%$

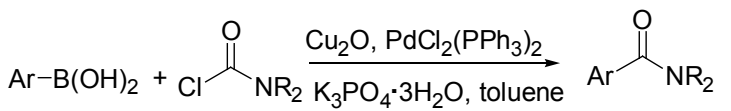

$$
\begin{aligned}
& \mathrm{R}^{1} \text { : alkyl } \\
& 7 \text { examples, yield } 60 \% \sim 93 \%
\end{aligned}
$$

\section{Scheme 21}

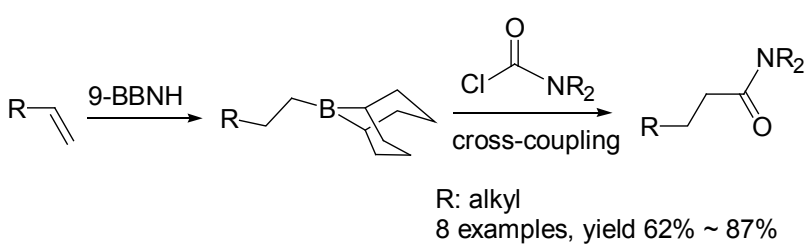

Scheme 22

2008 年 Sugihara 等 ${ }^{[57]}$ 通过铱催化的碳氢活化从奥 制备了2-宷基硼酸酯，在钯催化条件下该硼酸酯可以与 氯甲酰胺偶联, 由此发展了一条合成具有潜在药理活性 的 2-取代-蒾衍生物的新路线 (Scheme 23).

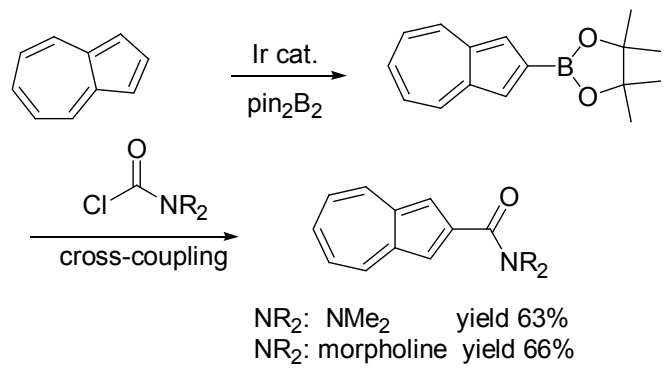

Scheme 23
2010 年 Herr 等 ${ }^{[58]}$ 报道了 $N$-甲基- $N$-甲氧基氯甲酰胺 与有机硼酸的交叉偶联反应，在乙醇溶剂中使用 $\mathrm{PdCl}_{2}\left(\mathrm{PPh}_{3}\right)_{2}$ 作为催化剂, $\mathrm{K}_{3} \mathrm{PO}_{4} \cdot 3 \mathrm{H}_{2} \mathrm{O}$ 作为碱就可以将 芳基硼酸或烯基硼酸与 $N$-甲基- $N$-甲氧基氯甲酰胺偶联 得到相应的 Weinreb 酰胺 (Eq. 11).

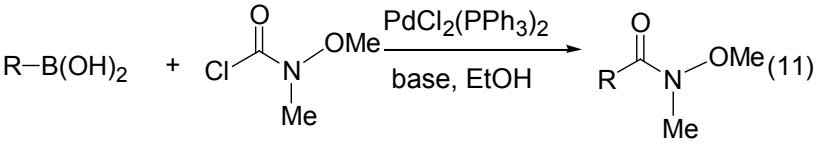

$$
\begin{aligned}
& \text { R: aryl, heteroaryl, alkenyl } \\
& 15 \text { examples, yield } 52 \% \text { 95\% }
\end{aligned}
$$

\section{5 有机硼化物与羧酸 “活性酯” 的交叉偶联反 应}

羧酸酯化合物中，酯基的活化能较高而不能直接作 为亲电试剂; 而当使用含有杂原子的羧酸吡啶酯、羧酸 三嗪酯和三氟乙酸酯作为亲电试剂交叉偶联反应可以 顺利进行.

2001 年 Yamamoto 等 ${ }^{[59]}$ 报道了 $\mathrm{Pd}(\mathrm{OAc})_{2}$ 催化三氟 乙酸苯酯与芳基硼酸的交叉偶联反应，在无碱加入的条 件下可偶联得到芳基酮类化合物. 使用呋喃基嗍酸和空 间位阻较大的芳基嗍酸时该反应未获得相应产物(Eq. 12).

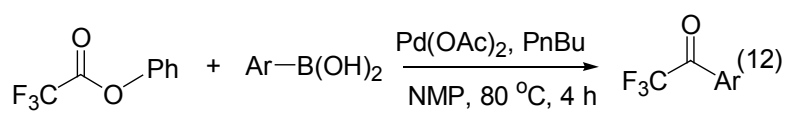

10 examples, $17 \% \sim 84 \%$ yield

2004 年 Chatani 等 ${ }^{[60]}$ 报道了羧酸吡啶酯与苯基硼酸 的偶联反应，在较温和的条件下即可偶联得到一系列苯 甲酮类化合物. 苯环上连有吸电子基或推电子基的芳基 羧酸酯参与反应时均以优秀的收率得到产物，而当烷基 羧酸酯作为亲电试剂参与反应时可以中等的收率得到 相应的酮(Eq. 13). 作者在研究中还发现此反应中吡啶 环上的 $\mathrm{N}$ 原子是顺利发生偶联必不可少的因素.

$$
\begin{aligned}
& \begin{array}{l}
\text { dioxane, } 50^{\circ} \mathrm{C}, 10 \mathrm{~h} \\
\text { Re aryl, alkyl } \\
10 \text { examples, yield } 68 \% \sim 97 \%
\end{array} \\
& \mathrm{Pd}(13)
\end{aligned}
$$

2011 年 Zhang 等 ${ }^{[61]}$ 报道了钯催化羧酸三嗪酯与有 机硼酸的交叉偶联反应，芳基羧酸三嗪酯可分别与芳 基、烯基及环丙基硼酸偶联，得到相应的芳基酮，脂肪 族䍨酸也可与芳基硼酸进行偶联制备相应的有机酮(Eq. 14). 其研究结果表明: 无碱条件下膦配体的用量对反 应收率影响显著, 当配体与钯的比例为 $2: 1$ 时, 偶联反 应收率最高. 


$$
\begin{aligned}
& \mathrm{R}^{1}=\text { aryl, alkenyl, cyclopropyl } \\
& \mathrm{R}^{2}=\text { aryl, alkenyl, alkyl } \\
& 26 \text { examples, yield } 15 \% \sim 99 \%
\end{aligned}
$$

\section{6 有机嗍化物与羧酸硫酯的交叉偶联反应}

2000 年 Srogl 和 Liebeskind 等 ${ }^{[62]}$ 报道了钯催化的 4卤丁基硫酯与芳基硼酸的交叉偶联反应，反应中利用钯 催化剂实现了碳硫键的氧化加成, 以中等到优秀的收率 得到芳基酮类偶联产物同时得到四氢噻吩. 在 4 -溴代丁 基硫酯的偶联反应中, 加入碘化钠会大大提高产物的收 率, 作者推测碘与底物中的溴发生的卤素交换促进了偶 联反应的顺利进行(Eq. 15).

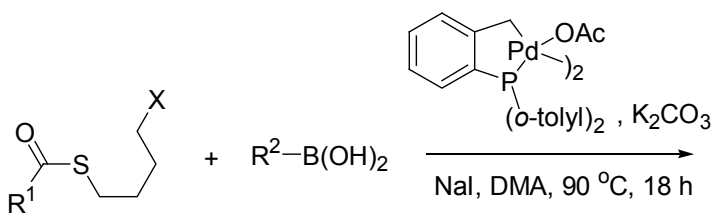

$$
R^{1} \stackrel{O}{\Lambda_{R^{2}}}+\square_{S}
$$

8 examples, $50 \% \sim 100 \%$ yield

2007 年 Liebeskind 等 ${ }^{[63]}$ 报道了钯催化手性氨基酸 硫酯与有机硼酸的偶联反应, 该反应以中等到优秀的收 率以及优秀的 $e e$ 值合成了手性 $\alpha$-氨基酮类化合物(Eq. 16). 值得注意的是作者优化得到了不加碱的偶联反应, 该方法有望应用于含有对碱敏感的多肽片段羧酸硫酯 的交叉偶联反应。

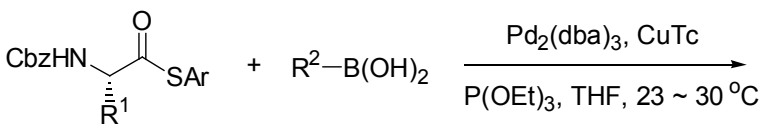

$$
\begin{aligned}
& \mathrm{CbzHN} \underbrace{\stackrel{\mathrm{O}}{\|} \mathrm{R}^{2}}_{\dot{\overline{\mathrm{R}}}^{1}} \\
& \text { yield } 48 \% \sim 99 \% \\
& \text { ee: } 99 \% \\
& \text { de: } 91 \% \text { } 99 \%
\end{aligned}
$$

2007 年 Liebeskind 等 ${ }^{[64]}$ 在合成鞘氨醇类化合物时 通过烷基羧酸硫酯与烯基硼酸的 Suzuki 偶联反应得到 $\alpha, \beta$-不饱和酮中间体, 该中间体经过三步反应得到了最 终产物 $($ - $)-D$-苏-鞘氨醇(Scheme 24).

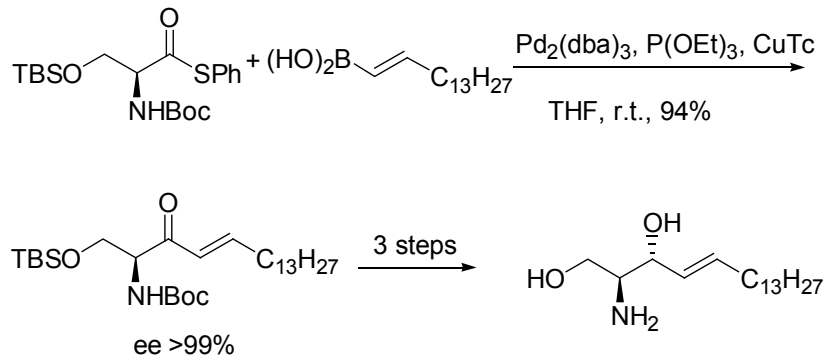

\section{Scheme 24}

2012 年 Prisinzano 等 ${ }^{[65]}$ 报道了精神作用药物 salvinorin A 衍生物的合成, 合成中利用了羧酸硫酯与 多种芳基嗍酸的交叉偶联反应，得到了多种 salvinorin $\mathrm{A}$ 衍生物(Eq. 17), 为该系列化合物的生理活性评价提 供了多种化合物.

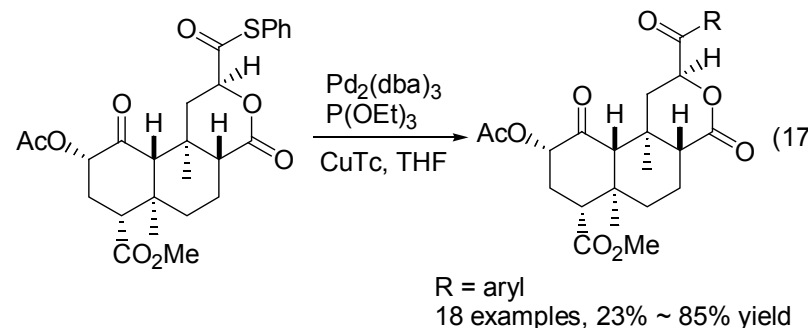

\section{7 结论与展望}

综上所述，多种有机硼化合物均可与酰氯、酸酐、 羧酸、氯甲酸衍生物、羧酸活性酯、羧酸硫酯等羧酸衍 生物发生偶联反应得到相应的偶联产物; 由于有机硼的 交叉偶联反应具有较优的官能团兼容性，这一反应已在 天然产物分子、生物活性分子的合成中已得到了实际的 应用. 由于该反应中作为亲核试剂的有机嗍化合物合成 简单，官能团兼容性好; 作为亲电试剂的羧酸衍生物来 源丰富、制备简单，这一钯催化有机嗍化合物与羧酸衍 生物的 Suzuki 偶联反应有望成为合成羰基化合物的重 要方法. 同时，一些不活泼羧酸衍生物特别是普通酯类 化合物和普通酰胺化合物由于氧化加成困难其与有机 硼试剂的偶联反应还是一个未解决的问题; 另外现有报 道的反应中所使用的有机硼化合物多限于芳基硼酸，对

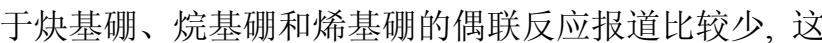
也值得有机化学工作者继续开展进一步研究.

\section{References}

[1] Hall, D. G. Boronic Acids, Wiley, Weinheim, Germany, 2005

[2] Dieter, R. K. Tetrahedron 1999, 55, 4177.

[3] Yamamoto, A.; Kakino, R.; Shimizu, I. Helv. Chim. Acta 2001, 84, 2996.

[4] Zapf, A. Angew. Chem., Int. Ed. 2003, 42, 5394.

[5] Xin, B.-W. J. Yulin College 2007, 17, 49 (in Chinese). (辛炳伟, 榆林学院学报, 2007, 17, 49.) 
[6] Negishi, E.; Chiu, K. W.; Yosida, T. J. Org. Chem. 1975, 40, 1676.

[7] Cho, C. S.; Itotani, K.; Uemura, S. J. Organomet. Chem. 1993, 443, 253.

[8] Bykov, V. V.; Korolev, D. N.; Bumagin, N. A. Russ. Chem. Bull. 1997, 46, 1631.

[9] (a) Bumagin, N. A.; Korolev, D. N. Tetrahedron Lett. 1999, 40, 3057.

(b) Bumagin, N. A.; Korolev, D. N. Russ. Chem. Bull., Int. Ed. 2004, 53, 364.

[10] Kabalka, G. W.; Malladi, R. R.; Tejedor, D.; Kelley, S. Tetrahedron Lett. 2000, 41, 999.

[11] Wang, J.-X.; Yang, Y.-H.; Wei, B.-G.; Hu, Y.-L.; Fu, Y. Bull. Chem. Soc. Jpn. 2002, 75, 1381.

[12] Gerard, J.; Hevesi, L. Tetrahedron 2004, 60, 367.

[13] Haddach, M.; McCarthy, J. R. Tetrahedron Lett. 1999, 40, 3109.

[14] Chen, H.; Deng, M.-Z. Org. Lett. 2000, 2, 12.

[15] Polackova, V.; Toma, S.; Augustingova, I. Tetrahedron 2006, 62, 11675.

[16] Urawa, Y.; Ogura, K. Tetrahedron Lett. 2003, 44, 271.

[17] Urawa, Y.; Nishiura, K.; Souda, S.; Ogura, K. Synthesis 2003, 2882.

[18] Bandgar, B. P.; Patil, A. V. Tetrahedron Lett. 2005, 46, 7627.

[19] Nishihara, Y.; Inoue, Y.; Fujisawa, M.; Takagi, K. Synlett 2005, 2309.

[20] Xin, B.-W.; Zhang, Y.-H.; Cheng, K. J. Org. Chem. 2006, 71, 5725.

[21] Ekoue-Kovi, K.; Xu, H.-H., Wolf, C. Tetrahedron Lett. 2008, 49, 5773.

[22] Zhang, L.; Wu, J.-L.; Shi, L.-J.; Xia, C.-G.; Li, F.-W. Tetrahedron Lett. 2011, 52, 3897.

[23] Martins, D. L.; Aguiar, L. C. S.; Antunes, O. A. C. J. Organomet. Chem. 2011, 696, 2845.

[24] Yu, A.-J., Shen, L., Cui, X.-L., Peng, D.-P., Wu, Y.-J. Tetrahedron 2012, 68, 2283.

[25] Chen, J.-X.; Peng, Y.; Liu, M.-C.; Ding, J.-C.; Su, W.-K.; Wu, H.-Y. Adv. Synth. Catal. 2012, 354, 2117.

[26] Eddarir, S.; Cotelle, N.; Bakkoura, Y.; Rolandoa, C. Tetrahedron Lett. 2003, 44, 5359.

[27] Lowe, J. A.; Drozda, S. E.; Fisher, K.; Strick, C.; Lebel, L.; Schmidt, C.; Hiller, D.; Zandi, K. S. Bioorg. Med. Chem. Lett. 2003, 13, 1291.

[28] Rahman, O.; Kihlberg, T.; Langstrom, B. Eur. J. Org. Chem. 2004, 474.

[29] Fujii, N.; Mallari, J. P.; Hansell, E. J.; Mackey, Z.; Doyle, P.; Zhou, Y.-M.; Gut, J.; Rosenthal, P. J.; McKerrowc, J. H.; Guy, R. K. Bioorg. Med. Chem. Lett. 2005, 15, 121.

[30] Mallar, J. P.; Shelat, A.; Kosinski, A.; Caffrey, C. R.; Connelly, M.; Zhu, F. Y.; McKerrowc, J. H.; Guy, R. K. Bioorg. Med. Chem. Lett. 2008, 18, 2883.

[31] Sessions, E. H.; Jacobi, P. A. Org. Lett. 2006, 8, 4125.

[32] Derbré, S.; Lecat-Guillet, N.; Pillon, F.; Ambroise, Y. Bioorg. Med. Chem. Lett. 2009, 19, 825.

[33] Chu, L.; Armstrong, H. M.; Chang, L.-L.; Cheng, A.-F.; Colwell, L.; Cui, J.; Evans, J; Galka, A.; Goulet, M. T.; Hayes, N.; Lo, J.; Menke, J.; Ok, H. O.; Ondeyka, D. L.; Patel, M.; Quaker, G. M.; Sings, H.; Witkin, S. L.; Zhao, A.; Ujjainwalla, F. Bioorg. Med. Chem. Lett. 2012, 22, 4133.

[34] Keenan, M.; Abbott, M. J.; Alexander, P. W. J. Med. Chem. 2012, $55,4189$.

[35] Yu, T.; Yang, J.; Wu, X.-Y. The 17th National Conference on
Organometallic Chemistry, Beijing, China, 2012, p. 93.

[36] Gooßen, L. J.; Ghosh, K. Angew. Chem., Int. Ed. 2001, 40, 3458.

[37] Frost, C. G.; Wadsworth, K. J. Chem. Commun. 2001, 2316.

[38] Gooßen, L. J.; Paetzold, J. Adv. Synth. Catal. 2004, 346, 1665.

[39] Kakino, R.; Yasumi, S., Shimizu, I., Yamamoto, A. Bull. Chem. Soc. Jpn. 2002, 75, 137.

[40] Goossen, L. J.; Koley, D.; Hermann, H. L.; Thiel, W. J. Am. Chem. Soc. 2005, 127, 11102.

[41] Goossen, L. J.; Koley, D.; Hermann, H. L.; Thiel, W. Organometallics 2006, 25, 54.

[42] Wang, J. X.; Zhang, Y. Q. Chin. Chem. Lett. 2004, 15, 641.

[43] Lim, K.-C.; Hong, Y.-T., Kim, S. Synlett 2006, 1851.

[44] Xin, B.-W.; Zhang, Y.-H.; Cheng, K. Synthesis 2007, 1970.

[45] Xin, B.-W. Synth. Commun. 2008, 38, 2826.

[46] Shen, X.-B., Gao, T.-T., Lu, J.-M. Shao, L.-X. Appl. Organometl. Chem. 2011, 25, 497.

[47] Lin, X.-F., Li, Y., Li, S.-Y., Xiao, Z.-K., Lu, J.-M. Tetrahedron 2012, 68, 5806.

[48] Gooßen, L. J.; Ghosh, K. Eur. J. Org. Chem. 2002, 3254.

[49] Gooßen, L. J.; Winkel, L.; Döhring, A.; Ghosh, K.; Paetzold, J. Synlett 2002, 1237.

[50] (a) Kakino, R.; Narahashi, H.; Shimizu, I.; Yamamoto, A. Bull. Chem. Soc. Jpn. 2002, 75, 1333.

(b) Kakino, R.; Narahashi, H.; Shimizu, I.; Yamamoto, A. Chem. Lett. 2001, 1242.

[51] Gooßen, L. J.; Ghosh, K. Chem. Commun. 2001, 2084.

[52] Kwon, Y.-B.; Choi, B.-R.; Lee, S.-H.; Seo, J.; Yoon, C.-M. Bull. Korean Chem. Soc. 2010, 31, 2672.

[53] (a) Dai, J.-J.; Liu, J.-H.; Luo, D.-F.; Liu, L. Chem. Commun. 2011, 47, 677 .

(b) Li, M.; Wang, C.; Fang, P.; Ge, H. Chem. Commun. 2011, 47, 6587.

(c) Li, M.; Wang, C.; Ge, H. Org. Lett. 2011, 13, 2062.

[54] Duan, Y.-Z.; Deng, M.-Z. Synlett 2005, 355.

[55] Lysen, M.; Kelleher, S.; Begtrup, M.; Kristensen, J. L. J. Org. Chem. 2005, 70, 5342.

[56] Yasui, Y.; Tsuchida, S.; Miyabe, H.; Takemoto, Y. J. Org. Chem. 2007, 72, 5898.

[57] Fujinaga, M.; Suetake, K.; Gyoji, K.; Murafuji, T.; Kurotobi, K.; Sugihara, Y. Synthesis 2008, 3745.

[58] Krishnamoorthy, R.; Lam, S. Q.; Manley, C. M.; Herr, R. J. J. Org. Chem. 2010, 75, 1251.

[59] Kakino, R.; Shimizu, I.; Yamamoto, A. Bull. Chem. Soc. Jpn. 2001, 74,371 .

[60] (a) Tatamidani, H.; Kakiuchi, F.; Chatani, N. Org. Lett. 2004, 6, 3579 .

(b) Tatamidani, H.; Yokota, K.; Kakiuchi, F.; Chatani, N. J. Org. Chem. 2004, 69, 5615.

[61] Zhang, W.-Q; Yang, J.; Deng, M.-Z. 8th Domestic Annual Conference of Applied Industrial Catalysis Technology, Xi'an, China, 2011, p. 329.

[62] Savarin, C.; Srogl, J.; Liebeskind, L. S. Org. Lett. 2000, 2, 3229.

[63] Yang, H.; Li, H.; Wittenberg, R.; Egi, M.; Huang, W.; Liebeskind, L. S. J. Am. Chem. Soc. 2007, 129, 1132.

[64] Yang, H.; Liebeskind, L. S. Org. Lett. 2007, 9, 2993.

[65] Lovell, K. M.; Vasiljevik, T.; Araya, J. J.; Lozama, A.; Prevatt-Smith, K. M.; Day, V. W.; Dersch, C. M.; Rothman, R. B.; Butelman, E. R.; Kreek, M. J.; Prisinzano, T. E. Bioorg. Med. Chem. 2012, 20, 3100. 